\title{
Culturas y creencias malentendidas dentro y fuera de la clase de $L 2$ para inmigrantes adultos
}

\author{
Elena Merino Jular, Universitat Pompeu Fabra, Barcelona, España
}

\section{Introducción}

Algunos problemas en la comunicación interpersonal cotidiana surgen porque ciertos aspectos de la interacción no se han interpretado correctamente (léxico, implícitos, elementos no verbales, etc.) o porque los interlocutores discrepan en sus opiniones, a veces ideológicas. Pensemos en las peleas en las relaciones intraculturales de pareja, padre-hijo, jefe-empleado, etc., provocadas por simples incomprensiones.

Actualmente, gracias al desarrollo de la tecnología y los medios de transporte el contacto internacional y el movimiento de personas por todo el mundo son hechos ineludibles, lo que además pone de manifiesto la necesidad de un tipo de comunicación más abierta y tolerante. Obviamente, el primer paso para establecer un contacto internacional es el uso de una lengua común, pero muchas veces ésta no es suficiente para que la comunicación sea exitosa (debido a los factores mencionados anteriormente-implícitos, ideologías, etc. - -). Ser miembro de una cultura o sociedad significa compartir una serie de marcos conceptuales y maneras de comunicar con el endogrupo que no tienen por qué coincidir con los de otros grupos. En ciudades multiculturales, como Barcelona donde la población extranjera representa un $18 \%{ }^{1}$ de la población total, fluyen diversas lenguas y culturas que dan pie a diferentes formas de vida, comunicación y relaciones interpersonales.

Debido al creciente interés en la comunicación intercultural y en la inmigración económica, este estudio ${ }^{2}$ se propone descubrir qué tipo de conflictos comunicativos pueden surgir entre personas de diferentes nacionalidades. Para alcanzar este objetivo se observarán los antecedentes del estudio, sobre todo en relación al aula, y las teorías que lo enmarcan, para presentar así un número limitado de resultados procedentes de los datos cualitativos recogidos. Finalmente, se analizarán dos ejemplos que den cuenta de la metodología aplicada. 


\section{Estado de la cuestión y justificación del estudio}

En las últimas décadas el campo de las segundas lenguas ha trabajado extensamente la interculturalidad en el aula. Miquel (1995), por ejemplo, hace una propuesta didáctica dirigida a la enseñanza de lengua extranjera (L2) ${ }^{3}$ para inmigrantes. En cuanto a los malentendidos, encontramos análisis comparativos entre la cultura española y otras (alemana, americana, japonesa, etc.) en los trabajos de Beyrich y Borowski (2000), Oliveras (2000, 2005) o Miquel y Sans (2004), cuyo objetivo no es otro que demostrar que cada cultura parte de unas convenciones diferentes. En cambio, los malentendidos con personas inmigradas, y en relación a la enseñanza de L2, han sido empíricamente menos trabajado.

En otros campos, como la antropología, sociología o psicología, también encontramos trabajos sobre malentendidos o conflictos culturales: Carroll (1988) hace una comparación antropológica entre la cultura americana y la francesa; el libro de Mayoral i Tor (2009) es un estudio sociológico donde los informantes, pertenecientes a cuatro nacionalidades distintas, narran los conflictos provocados por el desconocimiento sociocultural de la nueva cultura (C2); y Matsumoto, Leroux y Yoo (2005), sin embargo, parten de un enfoque psicológico para demostrar que los malentendidos interculturales producen emociones negativas.

Aunque existen algunos grupos, como el de CRIT (Comunicación y Relaciones Interculturales y Transculturales) de la Comunidad Valenciana, dedicados al análisis de la conversación entre inmigrantes y autóctonos, todavía necesitamos un modelo de análisis sistemático de los malentendidos tanto de la conversación propia-turnos, solapamientos, secuencias, etc., - (Hinnenkamp 2001) como del discurso en la interacción (interpretación semántica de las ideas, creencias e ideologías). De esta forma, podremos encontrar la manera más adecuada de averiguar cuáles son los conflictos que aparecen en la comunicación intercultural, y así aplicarlos a la enseñanza de L2 porque es aquí donde los recién llegados suelen tomar el primer contacto con la $\mathrm{C} 2$. 


\section{Objetivo}

Con el análisis de muestras concretas de interacción se intentarán desentramar las propiedades generales que rigen los procesos comunicativos entre personas de diferentes orígenes pero también de diferentes lenguas maternas, clase social, género y edad. Así, este proyecto tiene como objetivo primordial identificar los conflictos y malentendidos que se producen en la interacción entre algunas personas inmigradas y autóctonos de la sociedad española/catalana en la ciudad de Barcelona, para que más tarde los educadores puedan aplicarlo en sus clases.

En el punto de la investigación en el que nos encontramos, nos centraremos en los malentendidos que surgen en las entrevistas conducidas en inglés ${ }^{4}$ por la investigadora, una persona española, con 2 personas de Pakistán y otra de Bangladés. Concretamente, trataremos de:

1) describir cómo y por qué surgen malentendidos, y hasta qué punto se trata de malentendidos de tipo intercultural o ideológico.

2) analizar el efecto que pueden ejercer éstos (malentendidos e ideologías) en la comunicación interpersonal e intercultural.

Por su carácter interdisciplinar, para alcanzar los objetivos propuestos, tomaremos en cuenta diferentes perspectivas que giran en torno a la interacción: la competencia comunicativa intercultural, la enseñanza de L2 para inmigrantes, los malentendidos y las creencias, valores, actitudes e ideologías que inciden en la comunicación.

\section{Competencia comunicativa intercultural}

El esquema tradicional de la comunicación (emisor, receptor, código, mensaje, referente, canal y contexto) se olvida de que también tienen lugar otros elementos y procesos, por ejemplo, los procesos ostentivos e inferenciales, las expectativas, la intención, etc., (Escandell 2005) o el contexto ${ }^{5}$ (van Dijk). De esto se encargan disciplinas como el análisis del discurso o la pragmática, de constatar la existencia e importancia de otras competencias, que combinadas con la competencia lingüística, completan el acto comunicativo (Martín Rojo 2003). Es lo que Hymes (1971) llama la competencia comunicativa. El principio de cooperación de Grice, en 1975, y sus cuatro máximas (cantidad, calidad, relevancia y manera) establece que además los 
hablantes de una lengua controlan sus interacciones gracias a la colaboración que les permite inferir significados indirectos o implicaturas, es decir, significados no explicitados verbalmente pero que pueden llegar a inferirse.

Asimismo, en los años 80, Canale y Swain (1996) afirman que los mensajes se producen de acuerdo con las normas socioculturales que delimitan la forma adecuada de pronunciarlas. Según Escandell (2005) cada cultura establece una serie de parámetros diferentes para que la comunicación sea adecuada, dependiendo del objetivo (intención del emisor), la distancia social (pautas por las que se rigen las interacciones entre los interlocutores), la situación (grado de institucionalización de un intercambio) y el medio (oral o escrito). En otros campos, como la antropología lingüística, se cree en la indiscutible relación entre la lengua y los miembros de la sociedad que hablan esa lengua pues ésta define la manera de hacer o ver las cosas sobre conceptos supuestamente universales, como la cortesía (Duranti 2000).

En el aprendizaje y enseñanza de lenguas se incluye la competencia sociolingüística como un componente esencial para el completo dominio de la lengua, ya que hay elementos inherentes a la cultura que si no se saben desarrollar es como no haber aprendido una lengua por completo. En el MCER, Marco Común Europeo de Referencia (2001), concretamente en el punto 2.1.2, se describen las destrezas necesarias que un estudiante de una L2 debe desarrollar para alcanzar la competencia sociolingüística. Son, por tanto, las destrezas necesarias para la dimensión social de la lengua: normas de cortesía, normas que ordenan las relaciones entre generaciones, sexos, clases y grupos sociales, la codificación lingüística de determinados rituales fundamentales para el funcionamiento de una comunidad, etc.

Un nuevo documento para la enseñanza de las lenguas creado por el Consejo de Europa en 2008, el MAREP, Marco de Referencia para los Enfoques Plurales de las Lenguas y de las Culturas, tiene en cuenta la competencia intercultural, definida en Byram y Fleming (1998: 12) como "the acquisition of abilities to understand different ways of thinking and living, as they are embodied in the language to be learnt, and to reconcile or mediate between different modes present in any specific interaction”. Así, Schütz (1999) o Byram, Nichols y Stevens (2001) señalan que cuando una persona aprende una lengua extranjera, ésta se enfrenta a diferentes interpretaciones de los 
valores, normas, comportamientos y creencias que había adquirido en un primer momento como naturales o normales.

\section{L2 para inmigrantes adultos}

Es frecuente que los profesores de L2 para inmigrantes adultos actúen como mediadores interculturales, asistentes, amigos, o informadores. Es en estas clases donde se debería proporcionar al inmigrante con instrumentos que le orienten en la comunicación sociocultural e intercultural con los autóctonos puesto que los centros de lenguas son el primer lugar donde los recién llegados suelen acudir y porque el profesor supone un modelo de referencia sobre la nueva cultura.

En la enseñanza de L2 para inmigrantes adultos, a veces los profesores o bien son voluntarios, que pueden o no tener formación en la enseñanza de lenguas, o bien son profesionales de la lengua pero sin formación específica en la enseñanza de lenguas para este tipo de alumnado (Miquel 1995). En las clases de L2 para inmigrantes, no son pocos los inconvenientes que el profesor se encuentra: absentismo continuo, urgencia, alfabetos de caracteres diferentes, formación, materiales inadecuados, etc. Aún así, se han creado bastantes materiales dedicados a la enseñanza al alumnado inmigrante. Hernández y Villalba (2008), por ejemplo, han seleccionado una bibliografía muy completa donde se mencionan los ya reconocidos de Caja Madrid, la Cruz Roja, la Junta de Andalucía, etc. Otros autores dedicados a la enseñanza apuestan por el uso del malentendido como un elemento enriquecedor porque se concibe como una forma de obtener información sobre las maneras de comunicar y pensar de las culturas implicadas (Codó 1999; Oliveras 2000; Hinnenkamp 2001, Alonso 2006), no solamente para su aplicación en las relaciones sociales sino también para su uso en la clase de L2.

\section{Los conflictos comunicativos y malentendidos en las interacciones interculturales}

Ya hemos visto que cada interlocutor se acerca a la comunicación desde sus normas (culturales y conversacionales), valores, ideologías, poder, expectativas y creencias. Si estos aspectos difieren, el proceso comunicativo se rompe y pueden aparecer malentendidos (Oliveras 2000, 2005; Martín Rojo, 2003; Blas Nieves 2004). Pero antes 
es necesario señalar la dificultad que entraña el concepto de malentendido. Los conflictos que surgen en la interacción han recibido muchos nombres que, por otra parte, no son completamente sinónimos, aunque a veces la diferencia entre uno y otro no es tan clara. Algunas denominaciones en inglés, sobre todo por la extensa literatura en este idioma, de este fenómeno son: misunderstanding, non-understanding, miscommunication, awkward moments, inherent ambiguities, communication conflict, communication breakdown, commmunicative failure (Codó 1999; Hinnenkamp 2001).

Muchos autores afirman que si los interlocutores cuentan con un bagaje cultural diferente, es más probable que se produzca un fallo comunicativo, y éste repercuta en la imagen del otro (creación de estereotipos) pero a veces ocurre lo contrario; casi todas las sociedades cuentan con unos a priori sobre los otros y el hecho de que interactuemos con unas culturas determinadas sobre las que ya tenemos ideas preconcebidas obstaculiza la comunicación porque limitan nuestra mirada a la diferencia. Desde el punto de vista de la sociología, Schütz (1999) describe la situación en que se encuentra un forastero recién llegado a un nuevo lugar: con la incompatibilidad de esquemas entre el pensar habitual y los nuevos esquemas a los que se acerca. Hofstede (2005) define el choque cultural cuando una persona espera que las personas de su nuevo entorno social se comporten de igual modo que las de la cultura origen y se encuentra con que no es así. Sin embargo, hay que ser muy cauteloso para afirmar que un malentendido es producto de las diferencias culturales ya que, como vemos, son muchos los factores que entran en juego.

Hinnenkamp (2001) considera que los malentendidos son un evento con una estructura de reparación propia (corrective cycle). Esto demuestra el hecho de que los interlocutores han sido conscientes del conflicto y por eso intentan resolverlo. Por tanto, el malentendido se entenderá como un elemento de la interacción, que se interpreta y sobre lo que se puede discutir y negociar. Linell (1995, citado en Hinnenkamp 2001) establece siete tipos de malentendidos (M) que se explican a continuación y que usaremos para nuestro análisis aunque como veremos la diferenciación entre uno y otro no siempre es clara - $-\mathrm{y}$ esto es lo que pasa en la mayoría de las clasificaciones de malentendidos (House, Kasper y Ross 2003)—. M1 y M2 son malentendidos manifiestos (overt), M3, M4 y M5 encubiertos (covert) y M6 y M7 latentes (latent). 
M1: el malentendido se detecta y se repara inmediatamente para volver al momento comunicativo anterior al conflicto.

M2: el malentendido se detecta inmediatamente pero no se vuelve al momento anterior al conflicto sino que a partir de éste surge una nueva conversación.

M3: el malentendido se detecta gradualmente mediante signos de incoherencia, desviaciones (repeticiones, paráfrasis, circunloquios, etc.), inicios de reparación, inseguridad (verbal, no verbal, paralingüística) o momentos incómodos hasta que uno de los interlocutores se da cuenta de que se ha producido algún tipo de malentendido. Es entonces cuando se repara o no igual que en M1 y M2.

M4: presenta los mismos signos de incoherencia discursiva que M3, pero cuando se detecta, el malentendido no se gestiona como en los tipos M1 y M2, aunque sí se resuelve.

M5: Signos de incoherencia discursiva (M3 y M4) pero en este caso la conversación se para, disuelve, rompe o se reinicia con un cambio de tema.

M6 y M7: Debido a su carácter invisible (latente) M6 y M7 estos sólo podrán ser analizados en las narraciones posteriores al evento comunicativo. El malentendido M6 queda sin negociar porque no se considera como tal por los interlocutores, aunque más tarde se descubra. M7 se caracteriza por el sentimiento de incomprensión, pero ni es observable en la interacción ni se negocia.

Por otro lado, desde el punto de vista de la psicología, Matsumoto et al. (2005) mantienen que los sentimientos de incertidumbre y ambigüedad provocados por el malentendido magnifican el conflicto. Cuando una actividad comunicativa no es exitosa aparecen titubeos, interrupciones y reparaciones que crean sentimientos negativos o de rechazo porque entendemos que nuestro interlocutor no ha sabido interpretar nuestra intención comunicativa o no ha sabido responder adecuadamente a nuestros estímulos.

Finalmente, en el campo de aprendizaje de L2, y en concreto de Español como Lengua Extranjera (EL/E), expondremos el acercamiento, más culturalista, de Oliveras $(2000,2005)$ que parte de la narración de malentendidos sufridos por estudiantes de E/LE durante su estancia en España. Según Hinnenkamp (2001), éstos serían malentendidos de tipo latentes porque son conflictos que se narran ex post facto. El análisis Oliveras es importante porque permite conocer el origen de algunos malentendidos: el estilo comunicativo, la gestión de temas, el lenguaje no verbal o los 
valores, y las actitudes y acciones frente al contexto, espacio y tiempo. Seguidamente, reproducimos las preguntas que según esta autora han de hacerse para el análisis de malentendidos:

1. ¿Qué ha fallado en la comunicación?

- ¿Qué intención tenía el informante? (qué quería decir, qué imagen quería dar...);

- ¿Cómo se interpretó? (qué dijo en realidad, qué imagen dio)

2. El malentendido está relacionado con la diferencia cultural en la expresión y apreciación: del espacio, del tiempo, del contexto.

3. ¿Se podría haber evitado el malentendido? ¿Cómo? ¿Qué estrategias o habilidades podrían haberse utilizado?

4. Si se trata de una situación que no podía haberse evitado: ¿cómo reducirla para no dañar o 'recomponer' la imagen de la persona?

\section{Creencias, valores, actitudes e ideologías}

Para la interpretación de las interacciones comunicativas es necesario también analizar el significado del discurso producido, que además de las variables sociolingüísticas (edad, género, estatus social, etc.), está influido por las creencias culturales (valores, normas) e ideológicas de los individuos o, a la inversa, los discursos creados para el grupo social al que se pertenece influyen en el discurso personal, y otras prácticas sociales. Según explican la psicología cognitiva social (Bar-Tal 2000) o las teorías de van Dijk (2003, en prensa), las ideologías son sistemas de creencias compartidas que son útiles para los individuos porque se pueden aplicar a diferentes situaciones, eventos $\mathrm{y}$ acciones, y suelen crearse para y por el grupo siempre en situación de polarización con el otro.

Existe otro tipo de creencias que o bien se crean por la experiencia personal y subjetiva del individuo (almacenada en la memoria episódica, dentro de la memoria a largo plazo) o bien se interrelacionan con otras opiniones de individuos del mismo grupo ya que son controladas por una ideología compartida, pero con variaciones personales. Estos modelos mentales son necesarios para la producción e interpretación del discurso porque indican "how we personally plan, understand, interpret, experience and later remember all events and actions we are involved in" (van Dijk, en prensa). Asimismo, hay que tomar en consideración el contexto tradicional objetivo (situación, lugar, participantes, etc.) y el contexto subjetivo o construcción mental sobre nuestro interlocutor y la situación, es decir, mi modelo del conocimiento de los demás. ${ }^{6}$ 


\section{Metodología: la etnografía y el análisis del discurso}

La etnografía y el análisis del discurso se interrelacionan en cuanto que al estudiar a la gente participante de una cultura, se están describiendo sus puntos de vista, perspectivas y acciones. Para este estudio se observaron 15 clases de L2 (catalán y castellano) y se realizaron 11 entrevistas a dos personas de Pakistán y una de Bangladés. Sin embargo, no pudimos encontrar malentendidos en el aula debido a la orientación hacia la forma de algunas clases (es decir, la gramática), a la naturaleza invisible de algunos de estos conflictos y a la desigualdad de poder simbólico (Bourdieu 1985) dentro de un contexto institucional donde el profesor tiene la autoridad. Por este motivo, hasta el momento, solamente contamos con datos cualitativos extraídos de las entrevistas, es decir, la narración de los malentendidos por los que pasaron los informantes y los conflictos comunicativos observables en la interacción.

\section{Entrevistas}

Contamos con un corpus de 11 entrevistas, de las cuales 9 fueron grabadas y transcritas según la convención de símbolos en Tusón $(1997)^{7}$.

Los informantes de este estudio son 2 hombres de Pakistán, Alfonso (A) y Agustín (AG), de 36 y 44 años, respectivamente, y otro más joven, Gabriel (G) de 28, perteneciente a Bangladés, casi todos más o menos recién llegados, pues entendemos que el choque cultural es mayor en los primeros momentos de contacto con la nueva cultura (Hofstede 2010). Sin embargo, los tres informantes habían vivido en otros países antes de llegar a España (Tailandia, Italia, Inglaterra), lo cual inevitablemente influye en las percepciones, y actuaciones, que éstos puedan llegar a tener sobre los españoles y España.

Las entrevistas, semi-estructuradas, se realizaron en el período de marzo a agosto de 2009, con una duración media de 30 minutos con A y G y de 1 hora y 30 minutos con AG. El contenido, para todos, siempre giró en torno a temas relacionados con el pasado, presente y futuro, según la definición de García Parejo (2004) de inmigrante ${ }^{8}$, de los que surgieron conversaciones alrededor del trabajo, la familia, las relaciones interpersonales, etc. 
Brevemente, expondremos a continuación algunos de los resultados obtenidos en las entrevistas.

\section{Resultados generales}

El primer tipo de datos, las narraciones, se analizaron teniendo en cuenta las preguntas planteadas por Oliveras $(2000,2005)$ y desarrolladas en el marco teórico. Desafortunadamente, el número de datos extraídos de las narraciones es escaso puesto que, como ya se ha dicho, el malentendido puede ser un fenómeno invisible para los propios interlocutores. Sin embargo, describiremos las causas que provocaron malentendidos en algunas situaciones vividas por los informantes o sus compatriotas.

1. El idioma. Los malentendidos más frecuentes y visibles con los que los informantes dicen encontrarse es de tipo lingüístico, debido a su competencia en la L2: "I think if I speak with you in the Spanish language $\mid$ I will finish ten minutes $\mid$ it's better for me that you understand English" (AG) que, consecuentemente, determina el tipo de relaciones con la C2 (de poca duración, de tipo transaccional, etc.).

2. Relaciones entre el hombre y la mujer. Agustín relata toda la preparación previa que supuso la visita de una mujer, la investigadora. Era la primera vez que una mujer entraba en su casa y por esta razón tuvo que decírselo a todos sus amigos y vecindario (compuesto por pakistaníes y gitanos): "there were three women when we enter the house they were looking at me $\mid$ not you/this is the first time that one girl come with me in my house". Para AG, comunicarlo era importante porque "the women of my country welcome women/ no welcome to men $\backslash$ it's a difference".

2.1. Independencia de la mujer. AG reconoce el enfado del gobierno italiano al descubrir que una familia pakistaní que vivía en Italia había matado a su hija por querer casarse por amor. Sin embargo, éste defiende la normalidad de esta acción en su cultura: "why they kill $\backslash$ ? it's normal | why they killı?| because the girl do this in this family | if they don't kill the girl | perhaps the next year and the next coming years the other girl go out $\mid$ and they make marriage with their || $\mid$ they kill the girl for the family because the next time | any girl don't do the same as this girl".

2.2. Diferencias en los roles del hombre y la mujer. En una ocasión, Alfonso describe su sorpresa cuando una mujer tailandesa le prestó dinero porque, según ella, la mujer es la 
que lleva el dinero a casa: "I remember this and I will remember this whole life |because in my country ladies cannot make money| only men can make money".

3. Saludos. Gabriel explica la razón por la que algunas personas de su país no son competentes en la manera de saludar española. Lo atribuye a la falta de educación y piensa que la mejor forma de aprenderlo es habiendo vivido en otros países: "if I see somebody you have to say aSalaam 'Alaykum | it is our tradition |if somebody says that you need to reply Alaykum As-Salaam | but somebody doesn't do like this | that is why they are uncultured", "if somebody is illiterate they don't know what is hi".

4. Representación social del grupo. Los informantes comentan en alguna ocasión la imagen negativa de su grupo ante el mundo. Esta imagen provoca conflictos pero para contrarrestarla, se añaden características positivas: “they don't makes problems| that is one blame on us terrorista in the world |one blame Pakistani nation |not involve this Pakistani nation", "mostly people want of people want deal with peace and want to work you can see here too much Pakistani no person is thief | here ladrón |no ladrón Pakistani no/ they are good worker| they are good businessmen | and also very the morally good persons" (A).

\section{Tipología y causas}

Para los datos conversacionales extraídos de la interacción en las entrevistas expondremos las causas que provocaron los conflictos, de qué tipo son (manifiestos, encubiertos, o ambos) y cómo estos se pueden observar en el discurso (Hinnenkamp 2001).

Malentendidos manifiestos (overt): reconocimiento inmediato, reparación en la próxima oportunidad posible, y vuelta al estado anterior al malentendido. En la mayoría de los casos, sabemos que el malentendido ha sido reparado gracias a la confirmación del interlocutor: "now I get it", "I understand", etc.

1. El uso de metáforas. Según Lakoff y Johnson (1991:39) "la metáfora impregna la vida cotidiana, no solamente el lenguaje, sino también el pensamiento y la acción". Como las metáforas pueden diferir según la cultura que las crea, el reconocimiento de no haber entendido es inmediato y aunque se vuelve al estado anterior al malentendido, la reparación de éste suele durar más de un turno conversacional. 
2. Pronunciación o significado de las palabras. La incomprensión se debe a una pronunciación que el interlocutor no entiende y en algunos casos la reparación es más sencilla y rápida (traduciendo al castellano, repitiendo o poniendo ejemplos). Pero hay otros en los que el malentendido y se convierte en la raíz de una nueva conversación.

3. Uso del humor. A pesar de que los interlocutores mencionan explícitamente que algunos de los comentarios son una broma, la ausencia de las risas o las risas extemporáneas en el discurso indican que el otro interlocutor no se lo ha tomado como tal. El paréntesis provocado por la historia humorística provoca cambios de tema, a raíz de lo que se dice en la broma.

Malentendidos encubiertos (covert): los interlocutores los detectan debido a la incoherencia discursiva provocada por éste y se puede o no reparar como los anteriores.

1. Creencias e ideologías diferentes: cuando emergen creencias e ideologías contrapuestas, imbricadas en las opiniones de los interlocutores, como la religión, se produce una discontinuidad en el discurso que afecta al orden conversacional anterior.

1.1. Valores y normas: se mantienen como principios fuertes que hay que seguir, como los castigos, que normalmente también son ideas compartidas por un grupo, y el choque provoca cambios en la interacción.

2. Prejuicios: en algunas ocasiones lo que dice uno de los interlocutores se malinterpreta, incluso con el significado opuesto a lo que realmente se dice. La opinión está expresada correctamente pero que debido a los, suponemos, prejuicios de los interlocutores, provoca una inestabilidad en el discurso.

3. Desconocimiento del contexto: esto es lo que Gumperz (1995, citado en Hinnenkamp, 2001: 219) llama "lack of shared background knowledge". Los interlocutores relatan experiencias pasadas o tradiciones de su país, y debido al desconocimiento del interlocutor, aparecen interrupciones y se origina una nueva conversación en torno a esta nueva información.

¿Cómo se manifiesta en el discurso?

Cuando emerge un problema comunicativo, además de las violaciones de las máximas griceanas, hemos comprobado que la interacción se ve caracterizada por los siguientes fenómenos lingüísticos y paralingüísticos: interrupciones, solapamientos, cambios bruscos de tema, repeticiones, silencios, alargamientos vocálicos o 
consonánticos, respuestas contradictorias, duración variable de los turnos de palabra, silencios, pausas demasiado largas, enfatizaciones, titubeos, risas, etc.

\section{Algunos ejemplos}

Analizaremos un malentendido narrado por un informante (1), según el análisis de Oliveras $(2000,2005)$, y otro surgido en la interacción con la investigadora (2), según Hinnenkamp (2001).

(1) Una de las causas de incomprensión enunciada anteriormente es la diferencia en la concepción de los roles masculino y femenino. El malentendido ocurre en Tailandia, donde los participantes son Alfonso (A), hombre de Pakistán, y una mujer tailandesa (T). A le pide a $\mathrm{T}$ que le devuelva el dinero que le había prestado y se sorprende cuando T le responde: "you are man |you cannot make money |I am lady I can make money".

Lo que ha fallado es la expectativa que A tenía de T. A se acercó a T desde su contexto cultural pakistaní, en el que los hombres son los que trabajan. La respuesta de $\mathrm{T}$ confirma que su manera de concebir los roles masculinos y femeninos son diferentes a los de A y lo hace explícito. Esta explicitación es lo que sorprende a A y por eso, al terminar el relato, A afirma: "I remember this and I will remember this whole life |because in my country ladies cannot make money| only men can make money". Solamente se podría haber evitado el malentendido si A se hubiera informado previamente de quiénes son los actores activos del mercado laboral de Tailandia. Igualmente, A podría haberle explicado a T esta diferencia en Pakistán.

(2) Malentendido encubierto (covert):

Éste es un ejemplo compuesto por dos partes. En la segunda parte (2.2.) se intenta solucionar un malentendido que apareció en la primera (2.1), de una entrevista anterior. Gabriel (G) le cuenta a la investigadora (I) que ha leído dos libros, aunque I entiende "escrito" (wrote) en vez de "leído" (read).

G: and another thing |you know poetry |I used to read

I: $\mathrm{mhm}$

G: when I were in Bangladesh I: actually| I wrote two books (???)| in Bangladesh

I: and you are only twenty six/

G: no I am not of twenty six I'm =twen= 
$\mathrm{I}:=$ twenty $=$ five

En la segunda parte se comenta la situación anterior con la intención de obtener más información sobre esos dos libros.

I: but there's something you said/ that I'm interested about $\backslash$ it's a: you said you wrote two books/ in Bangladesh right?

G: wro:te? (con cara extraña)

I: or read $\backslash$ ?

G: read $\backslash$

I: $\mathrm{read} / \mathrm{ok} / \mathrm{I} \mathrm{sa} / \mathrm{ok} / \mathrm{ok} / \mathrm{I}$ thought you said I wrote/ ||| I don't know we were talking about::

$\mathrm{G}:=\mathrm{eh}:=$

I: =yeah= you don't re=member=

$\mathrm{G}:=$ what about $=\mathrm{it}$ ?

I: a:: I think we were talking about your life

G: about your life

I: in Bangladesh $\|$ and that you used to study| play sports and something about a book| like ah! like reading poetry

G: oh poetry =reading=

I: =yeah= and you said you read two books

$\mathrm{G}:=($ risas $)=$

$\mathrm{I}:=$ no wrote $=$

G: read read and read (pronunciando [red] [ri:d] [ri:d])

I: yeah yeah yeah (risas)

El malentendido se produce debido a una incomprensión fonética, y éste da lugar a un malentendido de contenido. En este ejemplo hay un reconocimiento gradual del malentendido: se producen interrupciones, solapamientos, signos de incoherencia, rodeos, y momentos incómodos, hasta que un interlocutor da cuenta del momento conflictivo, del que surge un tema relacionado - la poesía y el nombre de algunos autores bangladesíes y españoles.

\section{Conclusiones}

Con esta investigación hemos tratado de dar una visión general de la importancia del malentendido como un fenómeno frecuente pero a veces invisible para cualquier tipo de interacción, y en especial entre personas que parten de marcos conceptuales y culturales diferentes. Sin embargo, hemos comprobado la complejidad que entraña el concepto de 
malentendido y la precaución de aplicar la diferencia cultural como justificación para todos los conflictos comunicativos.

Debemos mencionar que los resultados no pretenden generalizar sobre culturas determinadas ya que es necesario tomar en cuenta el contexto objetivo y subjetivo comentado en el marco teórico y que puede incidir notablemente en la conversación.

Tras el análisis, hemos indicado las causas posibles de malentendido y hemos observado que algunos no son tan obvios, pero hay elementos en la interacción que lo indican: cambios de secuencias temáticas, interrupciones, titubeos, etc. Si el conflicto no apareciera explícito en la conversación, la interpretación podría parecer sesgada, interpretando erróneamente el malentendido

Finalmente, esta investigación sirve para constatar que la gestión del malentendido, es decir, el proceso de reparación puede producirse o no y puede ser más o menos inmediato, dependiendo de las causas que lo provocan.

Somos conscientes de las limitaciones del estudio, como la necesidad de recoger muestras de interacción con otras personas diferentes a la investigadora. Sin embargo, encontramos en éste un ejemplo valioso de situaciones de conflicto que pueden aportar las primeras pistas para su aplicación a la clase de L2 para inmigrantes, un campo relativamente nuevo en España.

\section{Referencias bibliográficas}

Alonso Cortés, T. (2006) El desarrollo de la competencia intercultural a través de los malentendidos culturales: una aplicación didáctica. Memoria de máster en Enseñanza de Español como Lengua Extranjera. Instituto Cervantes.

Bar-Tal, D. (2000) Shared Beliefs in a Society. Social Psychological Analysis. California: Sage.

Beyrich, D. y Borowski, C. (2000) Malentendidos interculturales en la comunicación cotidiana. Propuestas Interculturales. Actas de las VI Jornadas Internacionales TANDEM, pp. 41-48. Madrid: Edinumen.

Blas Nieves, A. (2004) La competencia sociocultural en la enseñanza de ELE a inmigrantes. Redele, 7. Revisado el 13 de junio de 2008 en $<$ http://www.educacion.es/redele/Biblioteca2007/AmeliaBlas.shtml $>$

Bourdieu, P. (1985) ¿Qué significa hablar? Economía de los intercambios lingüísticos. Madrid: Akal.

Byram, M. y Fleming, M. (eds.) (1998) Language Learning in Intercultural Perspective. Approaches through drama and ethnography. Introduction. Cambridge: Cambridge University Press. 
Byram, M. Nichols, A. y Stevens, D. (2001) Developing intercultural competence in practice. Clevendon: Multilingual Matters.

Canale, M . \& Swain, M. (1996) Fundamentos teóricos de los enfoques comunicativos. La enseñanza y la evaluación de una segunda lengua (I). Signos, Teoría y Práctica de la educación, 17: 54-62.

Carroll, R. (1988) Cultural misunderstandings. The French-American Experience. Chicago: The University Chicago Press.

Codó, E. (1999) Miscommunication in foreign language interaction: an empirical investigation. Working Papers, 109.

Consejo de Europa (2001) Marco común europeo de referencia para las lenguas: aprendizaje, enseñanza, evaluación. Instituto Cervantes. Revisado el 22 de febrero de 2008 en $<$ http://cvc.cervantes.es/obref/marco/presentacion.htm $>$

Consejo de Europa (2008) Marco de Referencia para los Enfoques Plurales de las Lenguas y de las Culturas. Austria: European Centre for Modern Languages.

Duranti, A. (2000) Antropología Lingüística. Madrid: Cambridge University Press.

Escandell, V. (2005) La Comunicación. Madrid: Gredos.

García Parejo, I. (2004) La enseñanza de español a inmigrantes adultos. Vademécum para la formación de profesores. Enseñar español como segunda lengua (L2) / Lengua extranjera (LE), pp. 1259-1277. Madrid: SGEL. Revisado en septiembre de 2008 en

$<\underline{\text { http://cvc.cervantes.es/obref/antologia didactica/claves/garcia parejo.htm }>}$

Grice, H.P. (1975) Logic and conversation. En: P. Cole y J.L. Morgan (eds.) Syntax and Semantics 3: Speech Acts, pp. 41-58, Nueva York: Academic Press.

Hernández, M. y Villalba, F. (eds.) (2008) Referencias bibliográficas sobre L2 e inmigración en España (I). Recursos para la investigación en adquisición / aprendizaje de lenguas, 2. Revisado en octubre de 2008 en $<\underline{\text { http://www.segundaslenguaseinmigracion.es/Investigaciones.html }>}$

Hinnenkamp, V. (2001) Constructing Misunderstanding as a Cultural Event. En: Di Luzio, A., Günter, S., y Orletti, F. (eds.) Culture in Communication. Analysis of intercultural situations, pp.211-243, Amsterdam: John Benjamins.

Hofstede, G. (2005) Viviremos nuestra vida en varias culturas diferentes. En Mondialogo. Intercultural dialogue Exchange. Revisado en febrero de 2010 en $<$ http://www.mondialogo.org/27.html? \&L=2>

House, J., Kasper, G., y Ross, S. (2003) Misunderstanding talk. En: House, J., Kasper, G., y Ross, S. (eds.) Misunderstanding in Social Life. Discourse approaches to problematic talk, pp. 1-21, Great Britain: Pearson Education.

Hymes, D.H. (1971) Acerca de la competencia comunicativa. En: Llobera, M. (coord.) Competencia comunicativa. Documentos básicos en la enseñanza de lenguas extranjeras, pp. 27-46, Madrid: Edelsa.

Lakoff, G. y Johnson, M. (1980) Metáforas de la vida cotidiana. Madrid: Cátedra, 1991.

Martín Rojo, L. (2003) Dimensiones principales en la comunicación intercultural. Educación y Futuro, 8, pp. 81-90.

Mayoral, D. y Tor, M. (2009) Cultures en interacció. La vida quotidiana. Barcelona: Edicions 62.

Matsumoto, D., Leroux, J. y Yoo, S.H. (2005) Emotion and intercultural communication. Kwansei Gakuin Sociology Department studies, 99: 15-38, 
Miquel, L. (1995) Reflexiones previas sobre la enseñanza de E/LE a inmigrantes y refugiados. Didáctica, 7: 241-254.

Miquel, S. y Sans, N. (2004) El componente cultural: un ingrediente más en las clases de lengua. Revista RedEle, 0.

Oliveras, A. (2000) Hacia la competencia intercultural en el aprendizaje de una lengua extranjera: estudio del choque cultural y los malentendidos. Madrid: Edinumen.

Oliveras, A. (2005) La competencia intercultural en el aprendizaje de una lengua extranjera: análisis de malentendidos culturales en el aula de ELE. Mosaico, 16: 24-26.

Schütz, A. (1999) El forastero. En: Estudios sobre teoría social, pp. 144-156, Buenos Aires: Amorrortu Editores.

Tusón, A. (1997) Análisis de la conversación. Barcelona: Ariel Practicum. van Dijk, T. (2003) Ideología y discurso. Barcelona: Ariel Lingüística.

van Dijk, T. (en prensa) Discourse and Ideology. En: Discourse Studies, London: SAGE.

\section{Referencia de la autora:}

Tras licenciarse en Filología Inglesa en el año 2003 por la Universidad de Valladolid, Elena Merino realizó un master en TESOL (Teaching English to Students of Other Languages) en la Universidad de West Virginia, Estados Unidos. En el 2007 hizo otro master en Lingüística y Aplicaciones Tecnológicas en la Universidad Pompeu Fabra, Barcelona, cuyo trabajo final ("The development of intercultural awareness and changes of beliefs: the effects of studying abroad on learners of English as a second language") fue dirigido por Carmen Pérez. Actualmente esta haciendo el doctorado en Comunicación Lingüística y Medicación Multilingüe en la UPF. Pertenece al grupo de investigación consolidado de Gr@el (Grup de Recerca d'Aprenentatge i Ensenyament de Llengües)

http://www.upf.edu/dtf/recerca/grups/grael/ y al Centro de Estudios del Discurso (CED:

http://www.discurs.org/es/presentacion/investigadores/). En estos momentos, esta desarrollando su tesis sobre los conflictos culturales con inmigrantes en relación a malentendidos que surgen en la comunicación dentro y fuera del aula de lengua extranjera.

Email: mariaelena.merino@upf.edu

1 Datos de Idescat (Estadística Oficial de Cataluña), 31 diciembre 2009: http://www.idescat.cat/es/ 2 Este trabajo forma parte del Gr@el - Grup de recerca sobre aprenentatge i ensenyament de llengües, de la Universitat Pompeu Fabra, que es un grupo de investigación consolidado y con financiación (Modalitat B, Agència de Gestió d'Ajuts Universitaris i de Recerca, Generalitat de Catalunya; núm. expedient: 2009 SGR 803), con Daniel Cassany como investigador principal (período 2009-2013).

3 Con lengua extranjera o L2 nos referimos a la enseñanza de la(s) lengua(s) que se usa(n) en el país receptor.

4 En la transcripción se mantuvieron los errores lingüísticos que aparecieron en el discurso. También, entendemos el problema que puede suponer en los resultados de la investigación usar y analizar una lingua franca.

5 T. van Dijk (comunicación personal, febrero de 2010). Para van Dijk, el contexto tiene que ver con la interrelación entre los interlocutores, es decir, con las representaciones mentales que cada hablante tiene sobre el otro y con la situación comunicativa propia.

6 T. van Dijk. (comunicación personal, marzo de 2010)

7 Código de transcripción aplicado (símbolos y significado):

| || Pausa breve o mediana. ? Entonación interrogativa. / entonación ascendente o descendente : :: alargamiento de un sonido. $\quad$ ! Entonación exclamativa. $\quad=\ldots . .=$ solapamiento en dos turnos:

(???) palabra ininteligible o dudosa ( ) elementos no verbales cursiva: palabras otros idiomas 
${ }^{8}$ Con inmigrante esta autora se refiere a una persona de diferente nacionalidad cuya presencia provoca cambios en la sociedad de acogida, aunque su paso por ésta sea transitorio. Es un no-ciudadano, que trabaja y pertenece a una minoría. Una persona que deja su país por causas económicas y políticas, generalmente, y que actúa guiado por un código lingüístico y cultural diferente al del país receptor, donde se integra más o menos dependiendo de la influencia de su país de origen. 